\title{
212 EFFECT OF EXERCISE TRAINING ON BONE MINERAL DENSITY IN POSTMENOPAUSAL WOMEN WITH BREAST CANCER
}

Reza Nuri, ${ }_{1}$ Arsalan Damirchi, ${ }^{1}$ Farhad Rahmani-Nia, ${ }^{1}$ Nader Rahnama, ${ }^{2}$ Hamid Emami ${ }^{1}{ }^{1}$ Department of Exercise Physiology, Faculty of Sport Sciences, University of Guilan, Rasht, Iran; ${ }^{2}$ Department of Exercise Physiology, Faculty of Sport Sciences, University of Isfahan, Isfahan, Iran; ${ }^{3}$ Department of Radiotherapy-Oncology, Isfahan University of Medical Sciences, Isfahan, Iran

10.1136/bjsm.2010.078725.212

Treatments for breast cancer such as adjuvant chemotherapy or hormone therapy can increase bone loss. Direct toxic effects of chemotherapy agents on bone formation cells as well as breast cancer itself may cause reductions of bone mineral density (BMD) in this population. Some studies reported that aerobic and resistance exercise improves bone health in preand postmenopausal women. Therefore, the aim of this study was to investigate the effect of exercise training on $\mathrm{BMD}$ in postmenopausal women with breast cancer. Thirty postmenopausal women with breast cancer $(58.27 \pm 6.31$ years $)$ who received surgery, chemotherapy and radiotherapy and were in current hormone therapy divided into two groups: intervention and control. Subjects of intervention group performed 15 weeks exercise training including walking (2 sessions per week) and resistance training (2 sessions per week different from walking days). Subjects of control group did not participate in any physical activity or exercise training. Body weight, body mass index (BMI), $\mathrm{VO}_{2}$ peak and lumbar spine and femur bone density was measured in two groups before and after of 15 weeks. Data analysed by using t test and covariance analysis. Results of this study indicated that after 15 weeks, there was significant difference between weight, $\mathrm{BMI}$ and $\mathrm{VO}_{2}$ peak in intervention and control groups $(p<0.05)$. Body weight and BMI decreased and $\mathrm{VO}_{2}$ peak increased in intervention group. There was no significant different between two groups in lumbar spine $(\mathrm{F}=2.98, \mathrm{p}=0.096)$ and femur $(\mathrm{F}=1.95, \mathrm{p}=0.174)$ $\mathrm{BMD}$ after 15 weeks. Body weight and BMI have protective effects on the BMD and the lower body weight and BMI, the lower BMD. Exercise training reduced body weight and BMI in subjects of this study, thus exercise training had no positive effect on BMD. Subjects in this study were in hormone therapy, and hormone therapy reduces BMD in breast cancer patients. It seems that hormone therapy has side effect on $\mathrm{BMD}$ in breast cancer survivor, and exercise training can not inhibit this effect. It can be concluded that the 15 weeks exercise training does no positive effect on $\mathrm{BMD}$ in postmenopausal women with breast cancer. 